Résumés des conférences et travaux

\title{
Histoire de la médecine
}

\section{Paléopathologie}

Pierre L. Thillaud

\section{(2) OpenEdition \\ 1 Journals}

Édition électronique

URL : https://journals.openedition.org/ashp/497

DOI : $10.4000 /$ ashp.497

ISSN : 1969-6310

Éditeur

Publications de l'École Pratique des Hautes Études

\section{Édition imprimée}

Date de publication : 1 octobre 2008

Pagination : 307-309

ISSN : 0766-0677

\section{Référence électronique}

Pierre L. Thillaud, «Paléopathologie », Annuaire de l'École pratique des hautes études (EPHE), Section des sciences historiques et philologiques [En ligne], 139 | 2008, mis en ligne le 26 novembre 2008, consulté le 12 juillet 2021. URL : http://journals.openedition.org/ashp/497; DOI : https://doi.org/10.4000/ashp 497 


\title{
PALÉOPATHOLOGIE
}

\author{
Chargé de conférences : M. Pierre L. ThiLlaud
}

Programme de l'année 2006-2007 : I. Paléopathologie, paléo-épidémiologie et pathocénoses. - II. Actualités paléopathologiques.

\section{Paléopathologie, paléo-épidémiologie et pathocénoses.}

Le concept de pathocénose a été défini en 1969, par Mirko Drazen Grmek (1924-2000), ancien directeur d'études de la section, comme l'ensemble formé par la coexistence de tous les états pathologiques qui règnent au sein d'une population déterminée dans le temps et dans l'espace et qui, dans une situation écologique stable, tend vers un état d'équilibre. Les changements diachroniques que ne manque de connaître cet équilibre, justifient l'étude de la dynamique de la pathocénose. Les relations qui unissent ces maladies ne relèvent que de trois modes fondamentaux : la symbiose, l'antagonisme et l'indifférence. De manière assez surprenante, M. D. Grmek ne s'est pas véritablement engagé dans l'application de cette approche séduisante de l'histoire des maladies et des états sanitaires des populations anciennes ou disparues. Quarante ans plus tard, que doit-on penser du concept de pathocénose? Deux choses, au moins. Si le terme fut régulièrement appliqué à des descriptions d'ensembles morbides affectant des populations anciennes, jamais ces exercices ne se soumirent aux exigences méthodologiques de son concepteur. Les progrès réalisés par la paléopathologie nous engagent à examiner les aménagements qu'il serait souhaitable de lui apporter pour le rendre plus opérationnel.

Les sources de la connaissance des pathocénoses historiques sont diversement alimentées par la documentation historique traditionnelle, les enseignements de la géographie médicale, les données issues des recherches paléodémographiques et au premier chef par la paléopathologie. Depuis près de 40 ans, la promotion de la paléopathologie est fondée sur son importante participation à la connaissance de l'histoire des maladies et sa contribution majeure dans la reconstitution des pathocénoses du passé, n'hésitant point aussi à prétendre apporter aux historiens les éléments probants de la nature des facteurs qui les déterminent et de bon nombre de ceux qui sont à l'origine de leur dynamique. Les résultats enregistrés par la paléopathologie organique, l'ostéo-archéologie et dans une moindre mesure, l'iconodiagnostic, portent témoignage de la réalité de cette contribution.

La pathocénose regroupe l'ensemble des maladies et des autres circonstances de la morbidité humaine (accidents) qui coexistent au sein d'une population donnée, à un moment donné. Cet ensemble tend à un équilibre tant qu'un facteur ne modifie pas les rapports hiérarchiques qui président à cet état en favorisant la dominance d'une nouvelle maladie ou plus exactement, d'un groupe de maladies, au détriment de celui qui occupait jusque là cette position. Tout se passe comme si de tous temps les groupements humains se sont trouvés soumis à une météorologie morbide et à ses inévitables 
variations alternant le grand beau temps, la grisaille et les tempêtes. Il convient donc d'identifier les nuages morbides qui composent ce ciel de la morbidité. A cet effet, le chargé de conférences propose l'usage du concept de « nuages morbides », proche de celui de « complexe pathogène » de Max Sorre mais tenant compte du caractère rétrospectif de son objet. Sept nuages forment le ciel de cette météorologie sanitaire. Le nuage dégénératif (arthroses, ...), traumatique (fractures, blessures, ...), carentiel (rachitisme, scorbut, anémies, ...), infectieux (germes banaux ou spécifiques : lèpre, peste, tuberculose, tréponématoses; paludisme, ...), congénital (nanisme, luxation de la hanche, anémies, ...), néoplasique (tumeurs bénignes et cancers primitifs ou secondaires) et toxique (carie dentaire, goutte, hydrargyrisme, ...).

Les facteurs de rupture qui semblent commander la dynamique des pathocénoses peuvent, à l'image des nuages morbides, être répartis dans sept groupes : physiques (climats, régimes des eaux, ...), chimiques (pollutions, intoxications, ...), politiques (expansions pacifiques ou belliqueuses, ...), économiques (variations des modes de production ruraux, flux commerciaux, industrialisation, ...), sociaux (sédentarisation, urbanisation, ...), sanitaires (hygiène, asepsie, antisepsie, vaccinations, ...) et culturels (religions, ...).

Déjà, quelques grandes ruptures pathocénotiques ont été identifiées. On peut citer : la néolithisation, marquée par la sédentarisation et la pratique de l'élevage, qui exposeront désormais les hommes à la majorité des maladies infectieuses qu'ils subissent encore; la bascule mycobactérienne survenue entre le $\mathrm{VI}^{\mathrm{e}}$ et le $\mathrm{XIV}^{\mathrm{e}}$ siècle, détrônant la lèpre au bénéfice de la tuberculose avec la complicité des coups de boutoir récurrents portés par les épidémies de peste; la spectaculaire compétition des diverses composantes du groupe des tréponématoses engagée à l'occasion des premiers échanges entre l'Ancien et le Nouveau Monde et, bien plus proche de nos contemporains, les effets très perceptibles des relations qui unissent les maladies cardio-vasculaires et dégénératives, aux surplus agricoles et à la tertiarisation des sociétés occidentales.

Force est de reconnaître toutefois que ces illustrations de la dynamique de la pathocénose relèvent toutes d'une dimension macro-sanitaire et que les outils proposés jusqu'à présent ne produisent leurs effets que sur une échelle bien moindre, dans la mesure où ils ne s'appliquent qu'à l'évaluation sanitaire fragmentaire d'un territoire et d'un peuplement forcément très limités.

Dans la perspective de doter la connaissance des pathocénoses anciennes d'une méthode adaptée à la réalité des sources disponibles, le chargé de conférences s'est rapproché de Joël Coste, directeur d'études de la Section mais également professeur des universités-praticien hospitalier, spécialiste de biostatistique et d'informatique médicale, pour lui proposer une collaboration de recherche visant à la modélisation des résultats des observations ostéo-archéologiques propre à définir une typologie des pathocénoses anciennes et à son expérimentation sur quelques nécropoles représentatives des pratiques habituelles de l'archéologie, de l'anthropologie physique et de la paléopathologie.

\section{Actualités paléopathologiques}

Au titre des actualités paléopathologiques, le chargé de conférences a procédé comme les années précédentes, à la revue systématique des dernières livraisons des 
périodiques spécialisés : Paleopathology Newsletter (PPN) et International Journal of Osteoarchaeology (IJOA) et des actes de congrès récents, prenant prétexte de certains articles ou communications, pour développer une analyse historique et critique des sujets choisis.

Un développement particulier a été donné d'une part, aux lésions de cribra orbitalia et d'autre part, au taurodontisme.

La lésion de cribra orbitalia a été décrite pour la première fois en 1885 par H. Welcker. Depuis, cet état cribleux de l'os du toit de l'orbite suscite les interprétations étiopathogéniques les plus variées. Durant 40 ans cependant, la communauté des paléopathologistes s'est accordée à reconnaître dans les anémies et plus généralement dans les carences martiales, nutritionnelles ou infectieuses, les causes de l'hyperplasie médullaire qui serait seule responsable de cette lésion de cribra orbitalia. Une reprise critique des données relatives à cette question, fondée sur l'examen macroscopique des lésions, la compréhension rétrospective de leur genèse et la paléo-épidémiologie, nous invite à ne plus identifier cette lésion comme une improbable forme hyperplasique « universelle», mais à distinguer trois types lésionnels bien distincts de cribra orbitalia, relevant respectivement : de la variation anatomique, de l'ostéopériostite et de l'érosion corticale. Cette proposition oblige désormais les paléopathologistes à prendre la mesure d'une plus grande diversité d'étiologies dans l'interprétation rétrospective des différentes formes lésionnelles de cribra orbitalia.

Le taurodontisme est le nom donné à une malformation dentaire consistant en un allongement de la chambre pulpaire avec pour conséquence, une division très distale des racines. Parce qu'elle fut primitivement et assez systématiquement observée sur la dentition de spécimens néanderthaliens, indépendamment de leur sexe et de leur âge ainsi que du type de la dent affectée, cette anomalie fut dans un premier temps considérée comme un caractère morphologique propre à ce peuplement préhistorique. Son observation comparable, également significative, dans de nombreuses populations primitives et chez nos contemporains sains ou plus souvent atteints d'une maladie génétique (Trisomie 21, Klinefelter, ...), s'est bien vite opposée à cette interprétation. Si à l'heure actuelle, aucune des explications physiopathologiques et étiologiques proposées ne s'impose véritablement, il semble que les efforts de mastication produits durant la croissance avec des dents déciduales et le bruxisme, puissent être à l'origine du plus grand nombre des cas observables de cette malformation dentaire.

Comme chaque année, les auditeurs ont été conviés à une visite du musée Dupuytren afin de se familiariser avec l'observation macroscopique des lésions osseuses anciennes. À la même occasion, le chargé de conférences avait invité le professeur Françoise Bouchet, directeur du laboratoire de parasitologie de la faculté de pharmacie de Reims, pour une présentation de : La paléoparasitologie : ses principes, ses méthodes et ses applications. 